\title{
Los saberes y decisiones productivo- tecnológicas en la agricultura familiar pampeana: Un mecanismo de resistencia al modelo de agricultura industrial
}

\section{Lía Nora Tamagno}

Facultad de Ciencias Agrarias y Forestales, Universidad Nacional de La Plata, Argentina Itamagno@agro.unlp.edu.ar

\section{María José Iermanó}

CONICET, Argentina

mariajoseiermano@gmail.com

\section{Santiago Javier Sarandón}

Comisión de Investigaciones Científicas de la provincia de Buenos Aires. Facultad de Ciencias Agrarias y Forestales, Universidad Nacional de La Plata, Argentina sarandon@agro.unlp.edu.ar

Cita sugerida: Tamagno, L. N., Iermanó, M. J. y Sarandón, S. J.(2018). Los saberes y decisiones productivo-tecnológicas en la agricultura familiar pampeana: Un mecanismo de resistencia al modelo de agricultura industrial. Mundo Agrario, 19(42), e100. https://doi.org/10.24215/15155994e100

Recibido: 10 agosto 2017 - Aceptado: 17 abril 2018 - Publicado: 07 diciembre 2018

(c) (1)(2) Esta obra está bajo licencia Creative Commons Atribución-NoComercial-CompartirIgual 4.0 Internacional cc) $\mathrm{By}$. $\mathrm{http}$ ://creativecommons.org/licenses/by-nc-sa/4.0/deed.es_AR 


\title{
Los saberes y decisiones productivo-tecnológicas en la agricultura familiar pampeana: Un mecanismo de resistencia al modelo de agricultura industrial
}

\author{
Knowledge and productive-technological decisions in the pampean family farming: A mechanism of resistance to \\ the model of industrial agriculture
}

Lia Nora Tamagno

Universidad Nacional de La Plata, Argentina

ltamagno@agro.unlp.edu.ar

María José Iermanó

Consejo Nacional de Investigaciones Cientificas y Técnicas,

Argentina

mariajoseiermano@gmail.com

Santiago Javier Sarandón

Comisión de Investigaciones Cientificas de la provincia de Buenos Aires,

Argentina

sarandon@agro.unlp.edu.ar

\section{ReSUMEN:}

La agricultura industrial ha generado pérdida de diversidad cultural y ello se expresa en la tecnología de producción. Las decisiones tecnológico-productivas de los productores familiares pampeanos, mediadas por sus saberes y convicciones operan como mecanismos de adaptación y resistencia y aportan a la resiliencia de los agroecosistemas. Con el objetivo de indagar sobre las decisiones productivas se realizaron entrevistas semiestructuradas a productores familiares del sudeste de Buenos Aires. Se encontró que las diversas lógicas, saberes y convicciones que median en las decisiones productivas de estos productores familiares, determinan una resistencia al modelo dominante y aportan a la resiliencia del sistema.

Palabras clave: Tecnología, Lógicas productivas, Sustentabilidad, Diversidad cultural, Agroecología, Región pampeana.

\section{Abstract:}

Industrial agriculture has generated changes that mean a significant loss of cultural diversity and this is expressed in production technology. Productive decisions were analyzed in family farms in the southeast of the province of Buenos Aires in order to revalue practices and knowledge as a basis for the design of agro-ecological management strategies. Semi-structured interviews were conducted, in-depth research on production technology. Our results suggest that the diverse logics, knowledge and convictions that mediate the productive decisions of these producers, determine a resistance to the dominant model and contribute to the resilience of the system.

KEYWORDs: Technology, Productive logics, Sustainability, Cultural diversity, Agroecology, Pampeana region.

\section{INTRODUCCIÓN}

La agricultura moderna, también denominada industrial, es el modelo de producción dominante en la Argentina y otros países, y se basa en la simplificación de los sistemas, el uso de elevadas cantidades de insumos derivados del petróleo (combustibles, fertilizantes y pesticidas), maquinaria, y de genotipos "mejorados" de plantas y animales (Gliessman, 2001; Flores y Sarandón, 2014a; Cáceres, 2015). Gras (2013) denomina a este modelo agrario como agronegocio, y lo caracteriza por el empleo de biotecnologías, un intenso ritmo de innovación tecnológica, altos requerimientos de capital, participación creciente de capital financiero y reorganización del trabajo y de la producción. Las consecuencias negativas de este modo productivo en el 
mundo, en nuestro país y en la región pampeana en particular son múltiples: pérdida de diversidad biológica y cultural, reducción del número de cultivos (erosión génica), "sojización”, restricción de la oferta tecnológica, contaminación de alimentos, aire, agua y suelos, pérdida de capacidad productiva de los suelos y exclusión de los productores con menores recursos (Sarandón y Flores, 2014a).

El modelo tecnológico que impulsa la agricultura moderna se caracteriza por el incremento del uso de la siembra directa (fuertemente asociada a la aplicación de herbicidas), nuevos cultivares con alto potencial de rendimiento, cultivos transgénicos, maquinaria agrícola adaptada a la producción en gran escala, el incremento en el uso de agroquímicos y el desarrollo constante de nuevos productos (herbicidas, insecticidas y fungicidas). Además, dicho modelo plantea respuestas tecnológicas que deben ser consideradas ejemplos de lo que constituye un technological fix, es decir, tecnologías de efecto inmediato pero de efectividad discutible y que pueden generar nuevos problemas (Scott, 2011). Cáceres (2015 p. 23) refiere que se trata de "soluciones tecnológicas rápidas y 'sucias' del problema en cuestión”, ya que son tecnologías que focalizan la atención en las manifestaciones sintomáticas de un determinado problema, en lugar de elaborar estrategias que ataquen las causas estructurales que lo generan.

Estas nuevas tecnologías, junto con una aparente rentabilidad positiva en el corto plazo, determinaron un reemplazo de las producciones tradicionales por el cultivo de soja transgénica (Glycine max L.) (Aizen, Garibaldi y Dondo, 2009) y el proceso de “sojización" mencionado por Guibert, Grosso, Arbeletche y Bellini (2011). También fue desplazada la producción tradicional ganadera y muchos establecimientos mixtos se transformaron en exclusivamente agrícolas, en un proceso de agriculturización que se inició a mediados de los años 70 (Azcuy Ameghino y León, 2005). Como consecuencia de ello, se produjo la expansión de la frontera agrícola y la concentración productiva, lo que llevó a una disminución sistemática de los establecimientos de menor tamaño (Gras, 2013). Azcuy Ameghino (2013) señala la desaparición de casi la mitad de las explotaciones familiares, y considera a las pequeñas y medianas explotaciones agrarias víctimas de la concentración y sujetos de la resistencia. El desplazamiento de muchos productores se debió, entre otros aspectos, a la imposibilidad de adaptarse a estas nuevas tecnologías, especialmente aquellos de menores recursos o con menor capacidad de acceso a las mismas (Ottmann, Spiaggi, Renzi y Miretti, 2009).

El rol de las nuevas tecnologías, consideradas durante mucho tiempo buenas per se (Sarandón y Hang, 2002), ha sido muy importante en los procesos de cambio en los establecimientos familiares (Mikkelsen, 2005; López Castro, 2009). Estos cambios, en gran parte, fueron consecuencia de una restricción de la oferta tecnológica e imposición del know how del paquete tecnológico dominante por parte del sector industrial (Cáceres, 2013). En este escenario, algunos productores adoptaron completamente la lógica de la agricultura industrial y la especialización agraria, ampliando su escala y utilizando tecnología "de punta". Otros, sin embargo, desarrollaron una hibridación tecnológica de resistencia (Ottmann et al., 2009), adoptando el paquete tecnológico incompleto con una menor utilización de insumos (Marasas, Cap, De Luca, Pérez y Pérez, 2012) y optando por diferentes estrategias tecnológicas que les permitieron mantener sus sistemas funcionando (Bilello et al. 2011).

En los últimos años, ante la percepción del fracaso cada vez más evidente del modelo (Sarandón y Flores, 2014a), varias tecnologías se han puesto en discusión, sobre todo por estar basadas en insumos caros y peligrosos. Tal como señala el INTA (INTA, 2005), la tecnología intensiva de insumos y capital no siempre ha satisfecho la demanda del sector de la agricultura familiar (que representa aproximadamente un $70 \%$ de todos los productores del país). Como contrapartida, el manejo sustentable de los agroecosistemas desde la Agroecología plantea el reemplazo de la tecnología de insumos por tecnologías de procesos, en las que juegan un interesante rol los saberes localmente adaptados y diversas prácticas de manejo agronómico (Bonaudo et al., 2014). Se ha destacado que los conocimientos y saberes de los productores, así como las lógicas puestas en juego, determinan la toma de decisiones o el diseño de estrategias productivas ecológicamente adecuadas (Gargoloff, Bonicatto, Sarandón y Albaladejo, 2009; Sarandón, 2009). En este sentido, Cáceres, Silvetti, Sotto, Rebolledo y Crespo (1997) definen el concepto de innovación tecnológica como el resultado de la 
incorporación de las tecnologías que el productor toma del medio o adopción tecnológica (exotecnologías) y la incorporación de aquellas que han sido generadas por los mismos productores, a partir de un proceso de experimentación y adaptación (endotecnologías).

Los productores familiares de la región pampeana no son de tipo campesino sino de tipofarmer (Muzlera, 2011) y se caracterizan por autoexplotar su fuerza de trabajo, ser dueños de los medios de producción, y por la posibilidad de acumular capital y producir mercancías, vinculadas al mercado internacional (Muzlera, 2011). Sin embargo, las relaciones internas de estas explotaciones no están regidas por la lógica capitalista, sino por otros principios relacionados con la condición familiar (López Castro, 2009; Balsa y López Castro, 2011). En ellos, la racionalidad del productor/a es la que define, en gran parte, la toma de decisiones (Muzlera, 2011; Balsa, 2011; Salembier, Elverdin y Meynard, 2016; Iermanó, 2015).

En este sentido, los productores familiares adaptan las nuevas tecnologías a sus situaciones concretas y a las diversas coyunturas, y en ello inciden las actividades familiares realizadas en procura de conservar el establecimiento, disminuir el riesgo y mantener el modo de vida (Balsa, 2011; Janzen, 2011). Por lo tanto, la subjetividad de los productores es definitoria al momento de organizar el proceso productivo y decidir estrategias (Muzlera, 2011). De este modo, en los establecimientos de producción familiar pampeana han perdurado lógicas productivas tradicionales, muchas veces consideradas un signo de atraso, que conviven con las nuevas tecnologías. Salembier et al. (2016) consideran que entre los productores pampeanos existen sistemas que se diferencian del modelo dominante, y que ellos son alternativas productivas que pueden ser fuente de inspiración para el desarrollo de sistemas sustentables. Tal es el caso del establecimiento La Aurora en Benito Juarez, que se ha constituido en un verdadero "faro agroecológico" para muchos productores de la región (Cerdá, Sarandón y Flores, 2014). Esto es coincidente con lo que indica Bonillo (2005) acerca de la importancia de considerar los saberes populares de los campesinos como alternativas tecnológicas que pueden aportar al diseño de sistemas de base agroecológica.

A diferencia de los productores empresariales, que adoptaron el paquete tecnológico modificando el estilo de vida, los productores familiares han incorporado de manera variable diferentes aspectos relacionados con el modelo dominante (Iermanó, 2015). Del mismo modo, Tamagno, Iermanó y Sarandón (2015) señalan que los productores familiares de la región pampeana han adoptado la producción de soja por diferentes motivos, especialmente por razones de rentabilidad en el corto plazo, pero adaptándola a sus diferentes realidades productivas.

Si bien en la mayoría de los casos existe alguna resistencia, ya sea por cuestiones económicas o por elección personal, el modelo dominante se filtró en estos sistemas productivos (Ottmann et al., 2009; Tamagno, Iermanó, Sarandón, Perez, 2014; Cáceres, 2015). De este modo, en el sudeste bonaerense, gran parte de los establecimientos agropecuarios han incorporado tecnologías de insumos, en diferentes medidas, pero otros, en cambio, han evolucionado sin concentrarse en el objetivo de maximizar los rendimientos de los cultivos. En lugar de ello, buscaron lograr una alta eficiencia de todo el sistema de producción, con base en las llamadas tecnologías de proceso (Forján, 2016). Por otra parte, las diferentes estrategias desarrolladas por los productores familiares pampeanos, vinculadas con la diversificación productiva intrapredial y extrapredial (Balsa, 2008; López Castro, 2009), son consideradas de importancia para el desarrollo de las zonas rurales y para un manejo más sustentable de los recursos (Sili, 2005).

Aun así, el fuerte predominio de la adopción de nuevas tecnologías (exotecnologías) por sobre las generadas por la experiencia de los propios productores ocasiona una pérdida de diversidad cultural, porque el "saber hacer" de los agricultores ha quedado parcialmente relegado en sus prácticas productivas. Desde esta perspectiva, es importante considerar que el modelo dominante no reconoce ni valora la existencia de un saber local (entendido como la gama de conocimientos propios, de carácter empírico, transmitidos oralmente) entre los agricultores/as (Toledo, 2005), y que revalorizar esos saberes en los procesos de producción de conocimiento se confronta a la idea dominante de que se puede desarrollar un solo tipo de agricultura ("el mejor”) (Sarandón y Flores, 2014b). Los saberes de los agricultores/as, sus prácticas, sus lógicas, han sido 
considerados, en general, más como un atraso que dificulta la incorporación de los nuevos conocimientos y tecnologías, que como saberes localmente adaptados que han coevolucionado con el ambiente (Sarandón, 2016). De Sousa Santos (2011) señala que la monocultura del saber y el rigor del saber son modos de producción de "no existencia" muy poderosos, y que esta lógica también declara atrasado todo lo que es asimétrico en relación a lo moderno y valora lo global o universal en contraposición a lo local o particular.

Desde la Agroecología se entiende que la resiliencia o la capacidad de adaptación y/o recuperación frente a la ocurrencia de fenómenos ecológicos, sociales, culturales y ambientales (variabilidad climática) que perturban el sistema de producción (Álvarez-Salas, Polanco Echeverry y Rios Osorio, 2014) es uno de los atributos de los agroecosistemas sustentables (Sarandón, 2014) y, como tal, debe ser evaluado en los sistemas de producción, incluidos los familiares. Por otro lado, es importante considerar que la investigación agroecológica ha sido realizada, mayoritariamente, en sistemas de tipo campesino o de pequeña escala, y son pocas las herramientas o investigaciones sobre sistemas familiares pampeanos, más extensivos, en los que existen elementos que pueden aportar a una producción de base agroecológica (Ottmann et al., 2009; Tamagno et al., 2014; Iermanó, Sarandón, Tamagno, Maggio, 2015). Del mismo modo, Salembier et al. (2016) indican que las caracterizaciones de los sistemas productivos de la región pampeana no deben limitarse a la descripción de las prácticas, y que será la relación entre las prácticas, los motivos o el análisis de las lógicas agronómicas y las performances lo que podrá aportar a la construcción de alternativas al modelo dominante.

Por todo ello, se asume como hipótesis que las decisiones tecnológico-productivas de los productores familiares pampeanos, mediadas por sus convicciones y saberes, operan como mecanismos de adaptación y resistencia al modelo dominante, al tiempo que aportan a la resiliencia de los agroecosistemas, disminuyendo, de esta manera, la incertidumbre y los riesgos. El presente trabajo pretende indagar sobre las decisiones productivas y, en particular, sobre aspectos tecnológicos como la actividad ganadera, la siembra de soja, el uso de nuevos materiales genéticos, el uso de agroquímicos (pesticidas y fertilizantes), el tipo de laboreo de suelo y las siembras consociadas en establecimientos familiares del sudeste de la provincia de Buenos Aires. Todo ello con la finalidad de revalorizar estas prácticas y saberes e incorporarlos al diseño de estrategias de manejo agroecológico.

\section{Metodología}

Se realizó un estudio exploratorio con la técnica de estudio de caso (Mitchell, 1983), durante el año 2013, en establecimientos que pertenecen a la región sudeste de la provincia de Buenos Aires, correspondiente a la unidad geomorfológica de la Pampa Austral Interserrana (Borda y Forján, 2106). Dicha región es principalmente agrícola-ganadera con predominio de sistemas mixtos (Mikkelsen, 2005). Sin embargo, esto se ha modificado como consecuencia de la agriculturización, debido a un cambio en el uso del suelo en detrimento de la actividad ganadera. En este sentido, Viglizzo et al. (2011) reportaron en esta zona un aumento del área sembrada con cultivos anuales del $13 \%$ y una disminución de la superficie ganadera del $13 \%$ entre 1990 y 2005, y señalan que ello estuvo asociado, además, a una intensificación en ambas producciones.

Se trabajó con 5 establecimientos agropecuarios, localizados en el sudeste bonaerense, en los partidos de Benito Juárez, San Cayetano y Tres Arroyos (Figura 1), que contaron con la colaboración de informantes calificados (profesionales de INTA y asesores privados). Se estudiaron cuatro casos representativos de sistemas familiares mixtos, (Obschatko, 2009) y un caso representado por un establecimiento agrícola empresarial (Tabla 1). Para la selección de casos se utilizó el criterio de diferenciación entre productores familiares y empresariales, por considerarlo pertinente para analizar los cambios entre una agricultura empresarial simplificadora de los sistemas productivos y una agricultura familiar en cuya toma de decisiones intervienen parámetros extraeconómicos, pero se priorizó el criterio de preservación de la explotación y del modo de vida asociado a ella (Balsa, 2011). 
A los fines de identificar los casos familiares, se tomaron en cuenta los criterios establecidos por Balsa (2011), pero flexibilizados con los aportes de Muzlera (2011) en la variable que hace a la mano de obra familiar y a la forma en que se configura el equipo de trabajo. Asimismo, se ha dado importancia central a la racionalidad de este tipo de productores como variable de selección, por considerar que el componente identitario es esencial en este tipo social. En el caso empresarial seleccionado, el productor realiza solamente tareas de gestión y decide las actividades en función de la rentabilidad, con un marcado predominio del uso agrícola del suelo y el énfasis puesto en la productividad y rentabilidad por unidad de superficie.

Debido a que la producción mixta es de mayor variabilidad y complejidad, se eligieron cuatro casos a los efectos de relevar mayor diversidad de situaciones. Si bien existen en cada uno de ellos ciertas particularidades, la historia de estos establecimientos es similar, ya que en todos los casos se trata de la tercera o cuarta generación dedicada a la producción agrícola-ganadera, por lo que vienen de tradiciones productivas mixtas similares y típicas de esta región. Por el contrario, debido a la simplificación de los sistemas agrícolas, se eligió sólo un caso que, por haber abandonado la ganadería, hace exclusivamente producción de granos. Este caso representa al universo de productores mixtos familiares que, por diversos factores derivados del nuevo modelo, se convirtieron en agrícolas empresariales.

TABLA 1

Caracterización de los productores

\begin{tabular}{|l|l|l|l|}
\hline Productor & Superficie (ha) & Actividades productivas actuales & $\begin{array}{l}\text { Tipificación } \\
\text { actual }\end{array}$ \\
\hline P1 & 650 & Mixto (agricultura, cría e invernada) & Familiar \\
\hline P2 & 447 & Mixto (agricultura, cría e invernada) & Familiar \\
\hline P3 & 415 & Mixto (agricultura e invernada) & Familiar \\
\hline P4 & 335 & Mixto (agricultura, cría e invernada) & Familiar \\
\hline P5 & 1550 (339 & Agricultura & Devenido en \\
empresarial
\end{tabular}

En cada caso estudiado se realizaron entrevistas semiestructuradas (Ander-egg, 1971), en dos oportunidades (mayo y diciembre de 2013), y se indagó en profundidad sobre la situación actual o existente, y sobre los diferentes aspectos en los que se centra el análisis. Se relevó información integral del sistema productivo, vinculada a la esfera productiva y socioeconómica. Se recolectaron los datos del planteo técnico de los cultivos y del manejo ganadero, buscando determinar, a través de preguntas orientadoras, en qué medida las decisiones técnico productivas responden a imposiciones del modelo de agricultura industrial o, por lo contrario, se sustentan en los saberes y/o convicciones de los productores. Para ello se privilegiaron algunos ejes relacionados a la tecnología de producción, que se consideran centrales en la difusión del modelo de producción dominante, tales como: relación ganadería-agricultura, modos de laboreo del suelo, siembra de soja y de otros cultivos, adopción de nuevos cultivares (transgénicos o no), uso de agroquímicos y siembras de cultivos consociados. Las entrevistas fueron desgrabadas en su totalidad y sistematizadas de acuerdo a los ejes seleccionados (Huberman y Miles, 1994; Fernández Núñez, 2006). El análisis e interpretación se centró en el material discursivo acumulado. Los dichos de los productores se presentan entre comillas y los comentarios de los autores se indican entre paréntesis. 


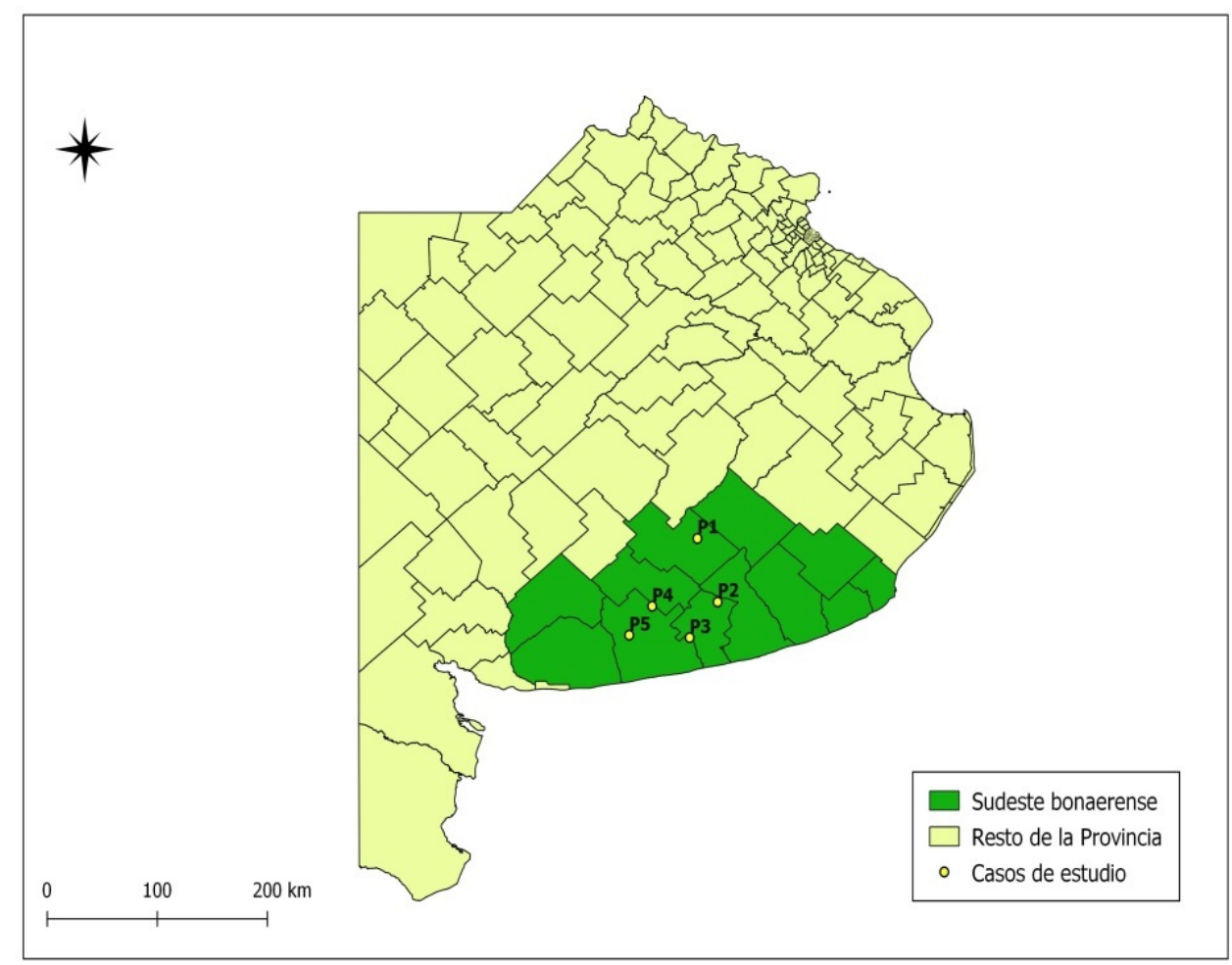

MAPA 1

Provincia de Buenos Aires con el área de estudio delimitada (sudeste de Buenos Aires) y la localización geográfica de los establecimientos.

\section{RESULTADOS Y DISCUSIÓN}

Se confirma que, en los últimos años, en los establecimientos familiares de la región pampeana se ha privilegiado la agricultura por sobre la ganadería, y la siembra de soja se ha difundido muy fuertemente, lo que demuestra el predominio del modelo del agronegocio en esta región. De este proceso dan cuenta todos los productores entrevistados, hecho que concuerda con lo mencionado por Gras (2013) y Cáceres (2015) en relación a dicho modelo.

Sin embargo, los productores entrevistados manifestaron niveles variables de adhesión y crítica al modelo. Todos los productores siembran soja, excepto el productor 1 (P1) y ello se debe a su convicción de que es posible un modo de producir más sustentable y saludable para el ser humano y el ambiente. Por este motivo, desde hace varios años viene transitando un proceso que le permitió llegar a producir carne y algo de granos, prácticamente sin uso de agroquímicos. Encontramos, en este caso, un ejemplo claro de resistencia de acuerdo a Azcuy Ameghino (2013). Este productor reconoce entre los impactos negativos del modelo la contaminación y daños en la salud por el uso de químicos y lamenta la desvalorización y la pérdida de saberes, cuando dice: "hoy en día está lleno de pooles; no hace falta el conocimiento del agricultor tradicional, que se va perdiendo...”.

Además, también aparece en el relato de los productores la percepción acerca de que la superficie de tierra necesaria para desarrollar la actividad ha aumentado como consecuencia del modelo. sí, el productor 4 (P4) dice: "Hoy con 400 ha no te alcanza para vivir; sólo mantenés el capital". Esto confirma que los productores son víctimas del modelo y conscientes de la amenaza por la pérdida sistemática de los establecimientos de menor tamaño ocurrida en los últimos años (Gras, 2013). 
Por otro lado, la gran demanda de uso de insumos que promueve el modelo dominante se pone de manifiesto, por ejemplo, cuando el productor 3 (P3) dice: "hoy se está echando mucha plata arriba de la hectárea (refiere al incremento de insumos utilizados)".

El productor agrícola (P5) adhiere completamente al modelo dominante y defiende el uso elevado de insumos químicos al decir: "Yo quiero seguir aumentando rendimientos (...) los gastos suman pero la producción y el precio multiplican", o "acá no tiene que haber nunca malezas". Sin embargo, este mismo productor también decide hacer maíz (Zea mayz L.) en su campo, en un momento en el que pocos elegían esa opción (debido a los altos costos de implantación de este cultivo) por reconocerle varias ventajas y aportes para el suelo. Con sus palabras - “[el maíz] fija carbono, deja cobertura, es lindo para cultivarlo, te llena el ojo (...) es el cultivo para el que tengo menos explicaciones”- demuestra que mantener la fertilidad del suelo a través de la inclusión de cereales como el maíz es un motivo que justifica sus prácticas productivas -tal como lo señalan Salambier et al (2016) -, aun cuando pueda considerarse menos rentable o requerir más inversión por su alto costo. Por otro lado, reconoce la presión ejercida por el modelo, porque, a pesar de adherir al mismo, el productor siente que cuando elige sembrar maíz en esas circunstancias de desventaja económica, suele ser considerado poco racional y debe dar explicaciones.

A pesar de que las nuevas tecnologías (semillas, insumos químicos, maquinarias, etc.) son entendidas, en general, como un avance, y buenas per se (Sarandón y Hang, 2002), se observó en las entrevistas la existencia de una clara conciencia del impacto negativo de la producción actual. Así P3 dice: "seguramente en este momento se están degradando los suelos (...) Muchos saben, el problema es que no te cierran los números, es un problema político. A mí me encantaría producir orgánico (se refiere a producir sin uso de químicos)”. Pero, también apareció, a favor del modelo, el argumento de la necesidad de producir más alimentos frente al aumento de la población mundial, mandato que opera sobre los productores, y que es frecuentemente utilizado como un eslogan del agronegocio, por ser nuestro país tradicional proveedor de alimentos para el mundo. En este sentido, agrega: "la población mundial aumenta (...) la tierra es la misma y la gente no", refiriendo a la necesidad de aumentar la productividad de los cultivos mediante el mayor uso de las tecnologías de insumos (semillas de mayor potencial de rendimiento, agroquímicos, maquinarias modernas). Por último, también los productores reconocieron la presión de las empresas para imponer el modelo. En relación a ello el productor 2 (P2) afirma: "en general, la gente va hacia el camino de cada vez hacer más y tener más, es siempre un furor para incorporar la tecnología (...) En eso las empresas hacen muy bien el marketing y todos nos enganchamos en eso, sobre todo en lo que es la agricultura, en la ganadería es un poco diferente”. Esto último reseña que la producción de granos involucra una mayor incorporación de tecnologías de insumos.

\section{Agricultura vs. ganAdería}

El desplazamiento de la ganadería por la agricultura, característica del proceso de agriculturización citado por Azcuy Ameghino y León (2005), no se observó en todos los sistemas evaluados. Sólo un productor (P5) abandonó completamente la ganadería en los años 90 y se define como productor empresarial: "El campo es $100 \%$ agrícola, pero no siempre fue así; se fue evolucionando"

Esta última expresión da cuenta, en primer lugar, de la adhesión al modelo, que ejerció una fuerte presión por el abandono de la ganadería extensiva por parte de los productores mixtos, y la idea de la necesidad de aumentar la producción de granos como sinónimo de evolución positiva de la empresa y del país y como aporte a resolver el hambre del mundo. En segundo lugar, confirma que las nuevas tecnologías son consideradas buenas per se, tal como lo señalan Sarandón y Hang (2002), y que el aumento de la producción de granos se alcanza a través de la incorporación de tecnologías "de punta" en el establecimiento. También señala la importancia que se le otorga a lo moderno respecto de lo atrasado, como señala de Sousa Santos (2011). Sin embargo, el mismo productor reconoce la presión externa en esa decisión cuando manifiesta: “a los finales de los 90 nos agarramos de la ganadería para salvarnos (...) dudamos bastante en vender la 
hacienda, pero otra era desaparecer o vender el campo". Así, para este productor, abandonar la ganadería y hacer una reconversión hacia la agricultura le significó subsistir en la producción y resistir a la desaparición que describen Azcuy Ameghino (2013) y Gras (2013), lo que coincide con lo mencionado por Mikkelsen (2005) para productores del sudeste bonaerense. El productor 3 también aporta a esta cuestión cuando dice: "Si la rentabilidad de la ganadería no caía, no se hubiera ido tanta gente del campo; la escuelita tenía 40 alumnos y hoy 7", lo que indirectamente indica que la ganadería genera la necesidad de que la familia resida en establecimiento. El efecto condicionante externo en el proceso de agriculturización se manifiesta también en los dichos del productor 2: "hoy hay muchos campos que se hacen agrícolas a la fuerza".

Los productores mixtos entrevistados se reconocen como familiares (además de caracterizarse como tales según las aproximaciones teóricas aquí consideradas), y mantienen la orientación tradicional de los establecimientos familiares pampeanos (Tsakoumagkos, 2009), aunque le otorgan a la ganadería niveles de importancia relativa diferentes. Se observa en estos casos que mantener la diversificación productiva ha sido la estrategia de subsistencia tal como lo refieren López Castro (2009) y Balsa (2011).

Los que se definen como ganaderos hacen de esta actividad productiva el centro de sus sistemas, sienten gusto por esa actividad y entienden a la agricultura como un aporte más a la producción, o un complemento financiero. Se reconoce, en este sentido, la rentabilidad económica que aporta la agricultura, pero entendida como complemento de una actividad más compleja como la ganadería, que ofrece menos riesgos en el corto plazo, debido a los ciclos más largos de producción, y que por lo tanto requiere plantear objetivos de mediano y largo plazo, hecho que concuerda con el análisis de Salembier et al. (2016). La elección de la ganadería aparece en varios relatos: "siempre me gustó la ganadería (...) para mi es la satisfacción más grande salir al campo y ver los animales contentos o llenos, gordos y no ver un suelo degradado (P1)..."; “yo no evalúo lo que me deja en carne (la ganadería) sino lo que me deja en la tierra" (P3); "uno sigue peleándola porque ama lo que hace y esta vida es lo que a uno lo mueve a seguir (...) esto es una filosofía de vida y la están matando" (P4); "si uno no tiene gusto por la ganadería, no la hace...” (P2). En estos relatos aparece la importancia del componente identitario referido por Balsa (2011) en la definición de la orientación ganadera de la producción, y se confirma el carácter definitorio de la subjetividad al momento de organizar el proceso productivo, tal cual lo señala Muzlera (2011).

Sin embargo, algunos de estos productores admiten que su gusto por la ganadería los coloca a contramano del modelo dominante, y asumen la descalificación que se instala sobre todo lo que se aparta de él. Así, el productor 3 afirma: "Soy un desastre, soy atípico (...) soy un fanático de la hacienda". Se observa, además, cierta conciencia de la resistencia al modelo que sugiere Azcuy Ameghino (2013).

Por otro lado, todos los productores entrevistados vinculan la ganadería con la necesidad de estar en el campo y de dedicar más tiempo a las tareas, en contraposición con la agricultura, especialmente simplificada en el marco del modelo hegemónico. En este sentido, el productor 2 dice: "la agricultura es mucho más sencilla (...) la ganadería es mucho más esclava. Uno tiene que estar".

Además, con este trabajo se lograron identificar prácticas que son una importante recuperación de saberes familiares. Por ejemplo, el hijo del productor 4, que es el encargado de la hacienda en ese establecimiento, cuando se refiere a la práctica de castración de terneros dice: "capo (en referencia a la castración) de grandes, echan más cuerpo y más kilos, a los $200 \mathrm{~kg}$, de parados (...) le pregunté al veterinario y me dijo que está de acuerdo (...) mi viejo y mi abuelo lo hacían así, me gustó que viene de una costumbre familiar”. En este relato aparece presente el saber local entendido como una gama de conocimientos propios, de carácter empírico, que son transmitidos oralmente entre los productores, tal como lo señala Toledo (2005), y también se observa cómo la subjetividad referida por Muzlera (2011) opera para mantener esas prácticas a pesar de la invisibilización que el modelo dominante impone.

La mayor complejidad de la ganadería (ciclos productivos más largos, diversidad de prácticas productivas, importancia de los saberes de los productores) puede explicar por qué el modelo de producción industrial no 
se ha filtrado tanto en la ganadería extensiva, hecho que permite considerar a esta actividad un fuerte factor de resistencia al modelo hegemónico.

\section{Producción de SOJA}

Todos los productores reconocen que la soja es una alternativa económico-financiera, lo que demuestra la influencia de la rentabilidad positiva en el corto plazo mencionada por Aizen et al. (2009) sobre las actividades productivas, incluso en los productores familiares. En este sentido, el productor 4 dice: "hoy la soja está subsidiando al resto", y "si tuviéramos que vivir como antes de la soja, no podríamos". Esto confirma la presión del modelo, que reduce las posibilidades de elección y presenta a la siembra de soja como casi la nica estrategia de permanencia en la actividad agropecuaria. Sin embargo, en los sistemas mixtos familiares, la superficie dedicada a la soja y la frecuencia de rotación es menor que en los sistemas agrícolas, en coincidencia con lo mencionado por Salembier et al. (2016). De esta manera, la siembra de soja en pequeñas superficies se presenta como un mecanismo de adaptación y resistencia al modelo dominante, ya que con sus ingresos permite de algn modo subsidiar la valorada actividad ganadera de los productores mixtos familiares.

Otro productor (P3) afirmó que sembrar soja le sirve "para limpiar los lotes después de las pasturas", lo que demuestra que da valor al aporte al control de malezas que este cultivo hace al sistema (por la aplicación de herbicidas totales). Sin embargo, la superficie sembrada de soja anualmente por este productor varía según los verdeos que siembra para alimentar la hacienda y los momentos en que compra los terneros. Toda la producción de soja es realizada con materiales genéticos resistentes a glifosato (RR), por la simplificación que significa en el manejo de las malezas, pero también porque las variedades tradicionales (no transgénicas) desaparecieron del mercado a los pocos años de difundirse la soja. Los primeros cultivares de soja RR aparecieron en el mercado de semillas de Argentina en el año 1996, y, para el año 2004, prácticamente el $100 \%$ de la superficie de soja en Argentina se cultivaba con variedades genéticamente modificadas (Rossi, 2006). Esto es un claro ejemplo de restricción de la oferta tecnológica e imposición del know how del paquete tecnológico que menciona Cáceres (2013), que limitó y limita las posibilidades de elección por parte de los productores y, además, determina la caracterización del modelo del agronegocio basado en el uso de biotecnologías que describen Gras (2013) y Cáceres (2015). Es importante destacar que este proceso no se hubiese dado en esta magnitud si el estado hubiera participado con alguna política de control o de limitación para la difusión de los nuevos materiales genéticos.

En este sentido, el productor 2 da cuenta de la presión que ejercen las empresas para imponer las nuevas tecnologías: "la llegada de la soja trajo otra variante (...) pero nos condiciona mucho en otros aspectos (...) es básicamente dependiente de tecnología, sobre todo de herbicidas y los insecticidas (...) uno queda muy cautivo de esos insumos y a su vez uno queda preso de las multinacionales".

Todos los productores entrevistados realizan la siembra en las fechas adecuadas para la región, aunque se encontraron diferencias entre los productores en relación a la distancia entre hileras. El productor 5 siembra soja a $17,5 \mathrm{~cm}$ entre hileras y dice: "sembrando a $30 \mathrm{~cm}$ y $30 \mathrm{~kg}$ por hectárea no te tiene que fallar nada. Yo no me animé (...) soy medio guaso; busco 10 o 12 plantas en el metro". Otro productor (P2) dice: "a mí me gusta sembrar a $21 \mathrm{~cm}$ porque cubre mejor el surco y controla mejor las malezas, se reparte mejor en el suelo". Estas diferencias dan cuenta nuevamente de la importancia de la subjetividad de los productores al momento de organizar el proceso productivo (Muzlera, 2011) y de la capacidad de adaptar el manejo del sistema (Salembier et al., 2016). Por otro lado, aportan diversidad biológica y cultural, lo que contribuye a una mayor sustentabilidad y resiliencia del agroecosistema. 


\section{OTROS CULTIVOS Y MATERIALES GENÉTICOS}

El desplazamiento de otros cultivos de verano (maíz y girasol Helianthus annuus L.) es una consecuencia de la sojización y fue referido por todos los productores entrevistados, quienes dieron cuenta de haber sembrado girasol en años anteriores (cultivo tradicionalmente importante en esta zona pampeana).

El productor 2 hasta el año 2004 producía girasol "hasta que apareció la soja transgénica (...) y barrió todo". Esto confirma lo expuesto por Aizen et al. (2009), cuando plantean tanto que la rentabilidad positiva en el corto plazo ha determinado un reemplazo de las producciones tradicionales por el nuevo cultivo, como que el modelo ejerce una fuerte presión sobre los productores.

El productor 3 dice: "el girasol es un lindo cultivo, hay que saber hacerlo, no como la soja; el girasol lo hace un productor, no lo hace un pool, hay que llenarse de tierra...”. Aquí se reconoce la simplificación que el cultivo de la soja ha impuesto, al no requerir del saber hacer del agricultor, lo que explica la producción de este cultivo en gran escala por grupos inversores o pooles de siembra y la consecuente caracterización del modelo actual como agricultura sin agricultores (Giardini, 2006). Por otro lado, señala claramente una valoración positiva hacia ese saber. La presión del modelo se ha manifestado a través de muchas formas, una de ellas es la restricción en la disponibilidad de semillas, debido a que las empresas dominan el mercado de éstas y de otros insumos, desde hace ya muchos años.

En este sentido, el productor 3 dice: "cuando aparecieron los híbridos de girasol las variedades casi salieron del mercado y desaparecieron años después (...) El hecho de que los girasoles sean híbridos hace que no puedas guardar sus semillas y quedás condicionado por la empresa que vende el híbrido". Si bien las decisiones de qué cultivos producir año a año están mediadas por diferentes circunstancias (precios de los granos, precios de los insumos y condiciones climáticas), se puede considerar que esta restricción es algo que los productores perciben, que no tienen modo de enfrentar y opera en contra de la diversificación productiva que cita López Castro (2013) como importante estrategia de permanencia de los productores familiares.

Los productores también reconocen que, a través de los años, se produjo un cambio en la disponibilidad de materiales genéticos de diferentes cultivos. Así, el productor 3 afirma: "los maíces actuales son buenos para producción de grano pero no tan buenos como los de antes para forraje”. Además, el productor 1 dice "cuando aparecieron los híbridos de girasol fue necesario empezar a usar químicos, y luego desaparecieron las variedades", lo que da cuenta del dominio de las empresas sobre los materiales genéticos y del objetivo productivista del mejoramiento genético que ha estado, además, asociado en las últimas décadas al alto uso de insumos, tal como lo señalan Sarandón y Flores (2014a).

Se destaca que los productores tradicionalmente guardan semillas de su propia producción para sembrar al año siguiente, lo que da cuenta de su capacidad de planificación y les confiere autonomía. Esta práctica de "uso propio", garantizada por la ley de semillas actual, pretende limitarse desde el año 2014 a través de una modificación de dicha ley. La mencionada reforma, promovida desde el año 2014 por las empresas semilleras, a partir de la aparición comercial de la soja Intacta RR2 PRO (doble transgénica de Monsanto), pretende que los productores paguen regalías extendidas por la semilla guardada. Se destaca que los sectores ligados a la producción familiar y de perspectiva agroecológica se vienen manifestando en contra de esta iniciativa, por considerar que profundiza la dominación de las semillas por parte de las empresas y atenta contra la producción soberana de alimentos.

\section{Uso DE AGRoQUímicos}

Uno de los aspectos que sin dudas caracteriza a estos sistemas industriales de producción es el elevado uso de pesticidas. En la Argentina, se pasó de un consumo de 73 millones de kg. / en 1995, a 317,17 millones de kg. / l en el año 2012 (CASAFE, 2013), lo que señala el éxito de esta tecnología, asociado a la búsqueda de mayores rendimientos. En este sentido, un solo productor (P1) se declara en contra del uso de agroquímicos y 
dice: "yo recibí esto por herencia y quiero también dejarlo en herencia a mi descendencia (...) en lo posible no quiero dejar un cadáver acá (...) tengo alergia a usar venenos”. De acuerdo a esta convicción, en la actualidad casi no usa insumos químicos, y a eso ha llegado a través de un proceso de transición de varios años. Ello demuestra, por un lado, que otro modo de producción es posible en establecimientos extensivos familiares pampeanos (Sarandón y Flores, 2014a; Iermanó, 2015), y, por el otro, que las convicciones y saberes de los productores operan como resistencia al modelo dominante y aportan diversidad productiva, fuente de resiliencia del sistema (Álvarez-Salas et al., 2014; Sarandón, 2014).

En el establecimiento que se dedica solamente a la agricultura (P5) es donde se aplican mayores cantidades de insumos químicos: cuatro aplicaciones de 2 litros por hectárea de glifosato además de otros principios activos, según aparición de resistencia en algunas malezas. El productor de ese establecimiento dice: "estos (refiriéndose a los herbicidas) están en el garaje todo el tiempo, para aplicarlos cuando haga falta. Porque estamos en ese proceso de resolver el problema de rama negra (Conyza bonariensis (L.) Cronq.) (una maleza que se ha vuelto resistente a glifosato). Aparece una ramita y hay que darle". Este productor lejos de cuestionar el uso elevado de agroquímicos que impone el modelo, lo naturaliza, aun cuando reconoce consecuencias de su uso indiscriminado, como la aparición de malezas resistentes.

Contrariamente, los otros productores hacen menor número de aplicaciones del herbicida con promedio de 2 litros por hectárea en el total del ciclo productivo, y plantean la preocupación por las fumigaciones excesivas: "nosotros usamos menos aplicaciones y muchas veces empatamos (alcanzan iguales rendimientos) con los de punta” (P3). Esto es coincidente con lo mencionado por Salembier et al. (2016). Se destaca de las entrevistas que el criterio de usar menos agroquímicos se relaciona especialmente con la necesidad de reducir los costos, como forma de mejorar el ingreso y mantenerse en la actividad, aunque aparece también la idea de impacto negativo que ellos producen sobre el ambiente y la salud humana. El menor uso de agroquímicos en los sistemas mixtos, con resultados productivos similares, podría responder a que la mayor agrobiodiversidad presente, como consecuencia de la actividad ganadera (ambientes seminaturales de los sistemas pastoriles) disminuye la necesidad de aplicaciones (Iermanó, 2015), lo que convierte a la ganadería en una estrategia tecnológico-productiva de adaptación que otorga mayor resiliencia al agroecosistema. Esta actividad también determina otra idea sobre el laboreo del suelo, lo que tiene influencia sobre el control de malezas y el menor uso de herbicidas.

Por otro lado, todos los productores refieren el problema de la aparición de las malezas resistentes al glifosato (hoy uno de los principales problemas de la agricultura argentina), como consecuencia de la gran difusión de la soja transgénica, al igual que lo mencionado por Salembier et al. (2016). Ello es un claro ejemplo de los technological fixes, de efecto inmediato pero de efectividad discutible (Rosner, 2004, citado en Cáceres 2013; Cáceres 2015), que pueden generar nuevos problemas (Scott, 2011), dado que en la actualidad el control de malezas en los cultivos resistentes a glifosato, debido a la aparición de malezas resistentes a este químico, requiere del uso creciente de otros herbicidas que el paquete tecnológico de la soja transgénica prometía abandonar.

\section{SIEMBRA DIRECTA VS. LABORES DEL SUELO}

Las prácticas de laboreo de suelo se han realizado tradicionalmente con numerosas herramientas y en diferentes momentos segn los cultivos. Para resolver los procesos de erosión del suelo que generaba el exceso de labores surgió, hace más de cuatro décadas, la tecnología de la siembra directa o labranza cero (INTA, 2013). Al estar asociada a un excesivo uso de herbicidas y fertilizantes (Rosenstein et al., 2007), la siembra directa ha sido un factor importante en la difusión del modelo dominante.

Entre los productores entrevistados se encontraron diferencias importantes en relación a estos aspectos tecnológicos. El productor agrícola (P5) es el único que hace toda la producción con la tecnología de siembra directa, lo que explica el mayor uso de herbicidas. Sin embargo, al preguntarle por la necesidad de uso de 
insumos químicos que impone la siembra directa dice: "se creció en glifosato, todo lo demás no". Además, hace referencia a que con el menor número de labores del suelo se gasta mucho menos en combustible: "es más caro pasar un escardillo (herramienta para control mecánico de malezas) que echar un twin pack (herbicida)”. En el mismo sentido el productor (P4) dice: "cuando apareció la directa la mirábamos con recelo. Y bueno, después el tema de los costos fue haciendo que fuéramos cambiando, porque uno de los insumos más grandes era el gasoil". Ello demuestra que la siembra directa también se fue imponiendo por una cuestión económicafinanciera.

Sin embargo, todos los productores son conscientes de que esta tecnología, si bien resuelve algunos problemas (erosión del suelo y altos costos de labores), no puede usarse tal como los promotores pretenden porque de su uso exclusivo derivan nuevas dificultades. En este sentido, la siembra directa puede considerarse también un ejemplo de technological fix, de acuerdo con Scott (2011) y Cáceres (2015). Esto se puede observar aún en los dichos del productor 5 cuando dice: "hay que ir al medio de los extremos; para AAPRESID (Asociación Argentina de productores en siembra directa) si sos de siembra directa es pecado mover el suelo (...) yo, hoy, cada 7-8 años hago laboreo a $12 \mathrm{~cm}$ en el suelo con disco para emparejar". Se percibe aquí cómo el modelo también avanza demonizando prácticas o herramientas de uso tradicional. Pero es importante destacar que, aun este productor que hace sólo agricultura y adhiere al modelo realiza labores de suelo, debido a que, desde su saber y experiencia, conoce las ventajas y aportes de la remoción del suelo en determinadas circunstancias.

El resto de los productores entrevistados usan una amplia gama de herramientas para labores de suelo, principalmente como consecuencia de la actividad ganadera. El productor 1, que decide evitar el uso de químicos, no hace prácticamente siembra directa y usa en general diversas maquinarias en la preparación del suelo para la siembra de pasturas y cultivos agrícolas. Además, refiere con claridad su idea del modelo dominante en relación a este aspecto: "con la siembra directa es difícil errarle, cualquier tonto puede tener éxito; pone los insumos recomendados y listo. En cambio acá hay que evaluar qué hacer y qué herramienta pasar (...) eso explica por qué hoy en día está lleno de pooles. No hace falta el conocimiento del agricultor tradicional, que se va perdiendo". Vuelve a aparecer aquí la simplificación del sistema que determina la pérdida de los saberes de los productores y de la diversidad cultural característicos del modelo de agricultura industrial.

En los sistemas mixtos, la actividad ganadera determina la necesidad de trabajar el suelo de manera particular. Estas decisiones tecnológicas se convierten en adaptaciones al modelo, y otorgan una mayor resiliencia al sistema. Así, el productor 3 dice: "laboreo (con referencia a las labranzas de suelo) cuando hay mucho pisoteo (por el paso de la hacienda por los lotes)".

En general, los productores utilizan diferentes sistemas de labranza (convencional, mínima o siembra directa) según las circunstancias, y seleccionan variadas herramientas que realizan diferentes remociones de suelo. En algunos casos ello determina el menor uso de herbicidas, tal como expresa el productor 4: "este año tuvimos suerte de hacer una sola pasada de glifosato porque disqueamos (pasaron el disco: herramienta que hace remoción parcial del suelo) en los lotes que venían de soja, y en los que venían de maíz también pasamos un cincel (herramienta de corte vertical para trabajar el suelo)". También son utilizadas labores mecánicas para controlar otras plagas que empezaron a ser muy importantes a partir de la difusión de la siembra directa, como los peludos (Chaetophractus villosus, Desmarest, 1804) o los bichos bolita (Armadillium vulgare, Latreille, 1804). Si bien los productores mixtos usan la herramienta de siembra directa, lo hacen adaptándola a las diferentes realidades productivas de acuerdo a su experimentación, lo que confirma la definición de endotecnologías que aporta Cáceres (1997). Por eso el productor 2 dice: "ahora tenemos la (siembra) directa, pero una directa loca”, refiriéndose a que lo hace a su manera. También subyace aquí la valorización negativa que el modelo impone a todo lo que se aparta de él, pero también se evidencia cierta resistencia a actuar completamente conforme al modelo, y, a pesar de esa valoración negativa, lo siguen haciendo porque le ven alguna utilidad. 
Por otro lado, se observó que la capacidad de realizar variados laboreos de suelo se asocia al uso de maquinarias propias y diversas. En este sentido, el productor 4 dice: "no tercerizamos nada, todo maquinas propias, todo de medio pelo pero al fin" Para estos productores tener sus propias herramientas significa aumentar su capacidad de autogestión y, de esta manera, reducir los riesgos e incertidumbres que genera la contratación de labores a terceros que, además de sumar costos de producción, no siempre pueden realizarse en tiempo y forma adecuadas.

Los productores mixtos valoran estas diferentes herramientas. El productor 2 dijo: "una vez usé cincel (...) cuando hice maíz (...) una herramienta casi obsoleta y me dio 7000 kilos (...) me preguntaron 'che, ¿qué semilla usaste?"”. La calificación de obsoleta de la herramienta sólo se puede entender en el marco de la imposición del modelo hegemónico, el que promueve que las nuevas maquinarias reemplacen totalmente las preexistentes, y la idea de que sólo con las nuevas tecnologías (semillas en este caso) pueden alcanzarse altos rendimientos de los cultivos. La prevalencia de lo moderno como un valor en sí mismo ha sido señalada como una característica distintiva de este modelo (de Sousa Santos, 2011; Sarandón, 2016).

Los productores mixtos entrevistados también demostraron tener una gran capacidad para adaptar las herramientas y un importante conocimiento de las ventajas del uso de cada una de ellas según situaciones (lotes, años, condiciones hídricas, etc.), lo que constituye un valioso saber que los productores familiares conservan, de acuerdo a Toledo (2005), y una importante estrategia de adaptación y resistencia. Las diferencias encontradas en relación al laboreo del suelo en los productores familiares determinan un importante aporte a la agrobiodiversidad cultural y a la resiliencia del sistema (Álvarez-Salas et al., 2014; Sarandón, 2014), y pueden considerarse un factor de resistencia en una zona donde la siembra directa se impone como un dogma, tal como lo señalan Salembier et al. (2016).

\section{SiEMBRAS CONSOCIADAS O INTERCULTIVOS}

La siembra de dos o más especies juntas en un mismo lote es considerada como una alternativa para un modelo más biodiverso y sustentable que el dominante (Dellepiane, Sánchez Vallduví y Tamagno, 2015; Sánchez Vallduví, 2012). Los más frecuentes en los sistemas mixtos son: pasturas perennes (mezcla de gramíneas y leguminosas) o cultivos anuales (para forraje o grano) consociados con leguminosas forrajeras. El productor 5 (que hace sólo agricultura) es el único de los entrevistados que no realiza este tipo de prácticas productivas, mientras que el resto de los productores hacen intercultivos según sus particularidades.

El productor 1 empezó a sembrar trigo (Triticum aestivum L.) consociado con trébol rojo (Trifolium pratense L.) en 1985, y en la actualidad siembra todos los cereales de invierno junto a este trébol, lo que depende del destino del cereal: "cuando la avena (Avena sativa L.) va para pastoreo no se siembra con trébol, porque compite mucho con lo de abajo, se cierra mucho en primavera”. En estos intercultivos, luego de la cosecha del cereal, el trébol rojo entra al circuito de la ganadería usado como forraje por tres años aproximadamente. Este mismo productor le reconoce a las leguminosas, además de la importancia como forraje, el valioso aporte de la fijación de nitrógeno (mineral de importancia en la nutrición de los cultivos y que el modelo dominante propone manejar a través de la aplicación de fertilizantes de síntesis química): "todo el mundo busca fijar nitrógeno con leguminosa y busca competir con malezas...”, y, además, dice: "yo tendría que empezar a usar el trébol blanco (Trifolim repens L.), que puede ser más competitivo que el rojo". Esto muestra la permanente búsqueda por adoptar nuevas prácticas o cultivos, que se adapten a su sistema y mejoren su sustentabilidad.

También el productor 2 menciona que el intercultivo ayuda en el manejo de plagas animales, cuando dice que siembra pasturas con avena y que "la avena (se siembra) como cultivo protector porque ayuda en el primer pastoreo"; "si hay pulgones prefieren la avena, entonces protegen un poco más a las gramíneas (de la pastura) que son más chiquitas." 
Las prácticas de intercultivo en los productores mixtos entrevistados demuestran también la importancia de los saberes sobre las prácticas y la capacidad de experimentación e innovación que desarrollan. Además, están muy vinculadas a la diversidad de herramientas que conservan y usan (algunas "obsoletas" desde la perspectiva del agronegocio), lo que les permite realizar tareas como la cosecha de las semillas de las consociaciones con hileradoras y cosechadoras propias.

\section{DisCUSIÓN FINAL}

La hibridación tecnológica es un mecanismo que permite la resistencia de los productores familiares de la región pampeana y la resiliencia de los agroecosistemas y se observa an en sistemas solamente agrícolas (P5). Esta estrategia de los productores de "filtrar", a través de sus saberes y lógicas, lo que es bueno para su situación y adaptarlo localmente puede ser visto como un sinónimo de atraso para el modelo dominante, pero, sin dudas, constituye un valor importante desde el punto de vista de la sustentabilidad.

De acuerdo a lo esperado, los productores mixtos familiares entrevistados se encuentran en una situación hibrida en cuanto a la adopción de tecnología, en concordancia con lo que plantea Ottmann et al. (2009), ya que han incorporado, de manera variable, diferentes aspectos relacionados con el modelo dominante (Iermanó, 2015).

A diferencia de la agricultura industrial, donde las técnicas se uniformaron y se simplificó el sistema a la siembra de unos pocos cultivos (de alto potencial de rendimiento) con alto uso de insumos externos, en los sistemas mixtos familiares se observa una mayor complejidad y diversidad de estrategias tecnológicoproductivas, tal como lo expresan Salembier et al. (2016) y López Castro (2009). Por lo tanto, si bien el modelo dominante se filtró, en la mayoría de los casos existe una resistencia determinada por los saberes y las convicciones de los productores, quienes, además, desarrollan la capacidad de experimentación local para entender no sólo los cultivos o el manejo del ganado, sino también las interacciones que ocurren dentro de sus sistemas diversificados (Bilello et al., 2011; Bonaudo et al., 2014).

Por otra parte, si bien se reconoce la importancia de las variables económicas, en estos sistemas familiares mixtos los objetivos de la unidad productiva y la toma de decisiones se rigen por elementos extraeconómicos (Balsa, 2011; Muzlera, 2011), y el vínculo con el establecimiento (o "el campo") es uno de los elementos centrales con los que se constituye la identidad de estos productores, para quienes el campo, además de ser la principal fuente de ingresos es un símbolo familiar (López Castro, 2009; Muzlera, 2011; Janzen 2011).

Estos productores familiares realizan sus actividades procurando conservar tanto el establecimiento y sus recursos naturales como el modo de vida familiar asociado a esas actividades, hecho por el cual adaptan el manejo del sistema a las diversas coyunturas (Balsa, 2011; Janzen 2011) poniendo en juego diversas estrategias de adaptación y resistencia (López Castro et al., 2009). Este trabajo pone esto en evidencia -y es un aporte para avanzar hacia el diseño de sistemas extensivos sustentables-identificando y rescatando lógicas y prácticas productivas con un valioso potencial para la construcción de estrategias agroecológicas que contribuyan a desarrollar un modelo productivo alternativo al de la agricultura industrial.

Si bien el trabajo se basa en el estudio de casos, se encuentran elementos que nos permiten aproximar algunas conclusiones:

- El relato de los productores da cuenta de la presión que ejerce el modelo dominante de agricultura industrial o agronegocio a través de restricción tecnológica y de la imposición de las nuevas tecnologías.

- Los saberes, gustos y convicciones de los productores familiares pampeanos rescatan las historias productivas de sus establecimientos y determinan la decisión de adoptar sólo en parte las nuevas tecnologías que se imponen, limitando parcialmente el avance del modelo de agricultura industrial. 
- Las diversas lógicas, saberes y convicciones que median en las decisiones productivas encontradas entre los productores familiares pampeanos, determinan una mayor agrobiodiversidad y aportan a la resiliencia del sistema.

- Estos productores deben considerarse sujetos de resistencia y actores imprescindibles en el diseño de un modelo de producción agropecuaria alternativo al del agronegocio en Argentina.

\section{Agradecimientos}

A todos los productores que nos abrieron las tranqueras de sus campos, compartieron generosamente sus experiencias y nos permitieron realizar este trabajo. Agradecemos el apoyo para realizar esta investigación proveniente del Programa de Becas de Investigación de la Universidad Nacional de la Plata (UNLP), del Proyecto de Incentivos a la Investigación de la UNLP (11/A273), de los Cursos de Agroecología y de Oleaginosas y CR de la Facultad de Ciencias Agrarias y Forestales de la UNLP, y del proyecto INTAAUDEAS-CONADEV (CIAC-940136).

\section{REFERENCIAS}

Aizen, M., Garibaldi, L. y Dondo, M. (2009). Expansión de la soja y diversidad de la agricultura argentina. Revista Ecología Austral. 19:45-54.

Álvarez-Salas, L.M., Polanco Echeverry, D.N. y Rios Osoirio, L. (2014). Reflexiones acerca de los aspectos epistemológicos de la agroecología. Cuadernos de desarrollo rural. Bogotá (Colombia) 11 (74): 55-74.

Ander-Egg, E. (1971). Introducción a las técnicas de investigación social. Editorial Humanitas. 2da. Edición. 335 pp.

Azcuy Ameghino, E. (2013). La cuestión agraria pampeana: de la lucha por las libertades capitalistas a la concentración económica (y siempre el latifundio). VIII Jornadas Interdisciplinarias de Estudios Agrarios y Agroindustriales, Buenos Aires, 29, 30, 31 de octubre y 1 de noviembre de 2013. CD Rom.

Azcuy Ameghino, E. y León C.A. (2005). La sojización: contradicciones, intereses y debates. Revista Interdisciplinaria de estudios agrarios. 23: 133-1157.

Balsa, J. y López Castro, N. (2011). La agricultura familiar "moderna”. Caracterización y complejidad de sus formas concretas en la región pampeana. En: López Castro, N. y G. Prividera (comps.) (2011). Repensar la agricultura familiar. Aportes para desentrañar la complejidad agraria pampeana. Buenos Aires: CICCUS Ediciones. p.45-76.

Balsa, J. (2008). “Cambios y continuidades en la agricultura pampeana entre 1937 y 2002. La zona agrícola del norte bonaerense”, en J. Balsa, G. Mateo y S. Ospital. Pasado y presente en el agro argentino. Buenos Aires: Lumiere, 2008. pp. 587 a 613.

Balsa, J. (2011). Notas para la caracterización de la agricultura familiar. En: VIIJornadas Interdisciplinarias de Estudios Agrarios y Agroindustriales, Buenos Aires, 1, 2, 3 y 4 de noviembre de 2011. CD Rom.

Bilello, G., .Pérez, R., Giordano, G. y Huinca, D. (2011). Productores ganaderos familiares y modernización. VII Jornadas Interdisciplinarias de Estudios Agrarios y Agroindustriales, Buenos Aires, 1, 2, 3 y 4 de noviembre de 2011. 18p. CD Rom.

Bonaudo, T., Burlamaqui Bendahan, A., Sabatier, R., Ryschawy, J., Bellon, S., Leger, F., Magda, D. y Tichit, M. (2014). Agroecological principles for the redesign of integrated crop-livestocksystems. Europ. J. Agronomy 57: 43-51.

Bonillo, M.C. (2005). Saberes campesinos, una estrategia para el desarrollo de tecnología apropiada para la agricultura orgánica realizada por agricultores familiares. Tesis de magister. Universidad de Temuco Chile. $131 \mathrm{p}$. Recuperado de: http://www.cedaf.fca.unju.edu.ar/sub/biblio/libros/tesis\%20saberes\%20campesinos\%20mari o\%20bonillo.pdf

Borda, M. y Forján, H. (2016) El área estudiada. En: INTA Ediciones. Rotaciones y secuencias de cultivos en la región mixta cerealera del centro-sur bonaerense. ISBN 978-987-521-699-0. Capítulo 1:p.10-12 
Cáceres, D. (2013). Tecnología agropecuaria y agronegocio. Un factor clave para la expansión del modelo agropecuario en Argentina. VIII Jornadas Interdisciplinarias de Estudios Agrarios y Agroindustriales, Buenos Aires, 29, 30, 31 de octubre y 1 de noviembre de 2013. CD Rom.

Cáceres, D. (2015 p23). Tecnología agropecuaria y agronegocios. La lógica subyacente del modelo tecnológico dominante. Mundo Agrario, 16 (31), abril 2015.30pp.

Cáceres, D., Silvetti, F., Ferrer, G., Soto, G. y Crespo, H. (1999). Lógicas productivas y prioridades tecnológicas de pequeños productores y técnicos que interactúan en un proyecto de desarrollo rural. Revista Cuadernos de Desarrollo Rural 43:81-95.

Cáceres, D., Silvetti, F., Sotto, G., Rebolledo, W., y Crespo, H. (1997). La adopción tecnológica en sistemas agropecuarios de pequeños productores. Agro sur, dic. 1997, vol.25, no.2, p.123-135.

CASAFE (2013). Cámara de Sanidad Agropecuaria y Fertilizantes. Mercado argentino de productos fitosanitarios / Año 2011 vs 2012. Disponible en: http://www.casafe.org/publicaciones/estadisticas/.Último acceso: marzo de 2016.

Cerdá EO, SJ Sarandón y CC Flores (2014) El caso de "La Aurora": un ejemplo de aplicación del enfoque agroecológico en sistemas extensivos del sudeste de la provincia de Buenos Aires, Benito Juárez, Argentina, en: SJ Sarandón y CC Flores, (Eds) La Agroecología: el enfoque necesario para una agricultura sustentable, Bases teóricas para el diseño y manejo de Agroecosistemas sustentables. Capítulo 16: 437-463. La Plata: Edulp Recuperado de http://s edici.unlp.edu.ar/handle/10915/37280

Dellepiane, A.V., Sánchez Vallduví, G.E. y Tamagno, L.N. (2015). Sustentabilidad del monocultivo e intercultivo de Helianthus annuusL. (girasol) con Trifolium pratense, Trifolium repens o Lotus corniculatus en La Plata, Argentina. Evaluación mediante indicadores. Revista de la Facultad de Agronomia, La Plata. 114 (Núm. Esp.1 Agricultura Familiar, Agroecología y Territorio): 85-94.

de Sousa Santos, B. (2011). Epistemologías del Sur. Utopía y Praxis Latinoamericana. Revista Internacional de Filosofía Iberoamericana y Teoría Social 54: 17-39.

Fernández Núñez, L. (2006). ¿Cómo analizar datos cualitativos? Fichas para investigadores. Butlletí La Recerca. Universitat de Barcelona, Institut de Ciències de l'Educació, Secció de Recerca. 13pp.

Flores, C.C. y Sarandón, S.J. (2014). La energía en los agroecosistemas. En: S.J. Sarandón y C.C. Flores (eds.) Agroecología: bases teóricas para el diseño y manejo de agroecosistemas sustentables. Capítulo 7: 190-210. La Plata: Edulp. Recuperado de http://sedici.unlp.edu.ar/handle/10915/37280.

Forján, H. (2016) Síntesis y conclusiones. En: INTA Ediciones. Rotaciones y secuencias de cultivos en la región mixta cerealera del centro-sur bonaerense. ISBN 978-987-521-699-0. Capítulo 9:p.69-74.

Gargoloff, N.A., Bonicatto, M.M., Sarandón, S.J. y Albadalejo, C. (2009). Análisis del Conocimiento y Manejo de la Agrobiodiversidad en Horticultores Capitalizados, Familiares y Orgánicos de La Plata, Argentina. Rev. Bras. de Agroecologia, 4(2). 4pp.

Giardini, H. (2006). Soja transgénica: agricultura sin agricultores. Recuperado de http://www.biodiversidadla.org/co ntent/view/full/28995

Gliessman, S. (2001). A energética dos agroecossistemas. Agroecología. Processos ecológicos em agricultura sustentable. Segunda Edición. Río Grande do Sul: Editora da Universidade. Cap.18: 509-538.

Gras, C. (2013). Expansión agrícola y agricultura empresarial. El caso argentino. Revista de Ciencias Sociales. DS-FCS. 26(32): 73-92.

Guibert, M., Grosso, S., Arbeletche, P. y7 Bellini, M.E. (2011). De Argentina a Uruguay: espacios y actores en una nueva lógica de producción agrícola. Pampa 07, Suplemento temático 2011:13-38.

Huberman, A.M. y Miles, M.B. (1994). Data management and analysis methods. En Denzin N.K. y Y.S. Lincon. Handbook of Qualitative Research. Thousand Oaks, CA: Sage. Pp. 428-444.

Iermanó, M.J. (2015). Sistemas mixtos familiares de agricultura y ganadería pastoril de la región pampeana: eficiencia en el uso de la energía y rol funcional de la agrodiversidad (Tesis doctoral). Recuperado de (http://sedici.unlp.e du.ar/handle/10915/46343). 
Iermanó, M.J., Sarandón, S.J., Tamagno, L.N. y Maggio, A.D. (2015). Evaluación de la agrobiodiversidad funcional como indicador del "potencial de regulación biótica" en agroecosistemas del sudeste bonaerense. Revista de la Facultad de Agronomia, La Plata. 114 (Núm. Esp.1 Agricultura Familiar, Agroecología y Territorio): 1-14.

INTA (Instituto Nacional de Tecnología Agropecuaria). (2005). Programa Nacional de Investigación y Desarrollo Tecnológico para la Pequeña Agricultura Familiar. Documento Base. Abril de 2005.

INTA (Instituto Nacional de Tecnología Agropecuaria) (2013), INTA Informa en web: http://intainforma.inta.go v.ar/?p=18029 visita $16 / 12 / 16$

Janzen, H.H. (2011). What place for livestock on a re-greening earth? Animal Feed Science and Technology, 166-167:783-796. doi:10.1016/j.anifeedsci.2011.04.055

López Castro, N. (2009). Cuando la persistencia es una cuestión de familia. Relaciones familiares, traspaso y género en explotaciones agropecuarias del sudoeste bonaerense (1987-2007). Mundo Agrario, 10(19): 32pp.

López Castro, N. (2013). Trayectorias de diferenciación social de la producción familiar pampeana en las últimas décadas. Estudio en el agro del sudoeste bonaerense (Puan y Adolfo Alsina, 1988-2012). VII Jornadas Interdisciplinarias de Estudios Agrarios y Agroindustriales. Buenos Aires, 1, 2, 3 y 4 de noviembre de 2011. CD rom.

Marasas, M., Cap, G., De Luca, L., Pérez, M. y Pérez, R. (2012). El camino de la transición agroecológica. Ciudad Autónoma de Buenos Aires: Ediciones INTA. 100p. Recuperado de http://inta.gob.ar/documentos/el-camin o-de-la-transicion-agroecologica.

Mikkelsen, C.A. (2005). Innovaciones tecnológicas productivas agrarias en el partido de San Cayetano: implicancias en la sostenibilidad del suelo. Mundo Agrario, 5(10).22p.

Mitchell, J.C. (1983). Case and situation análisis. The Sociological Review 31(2): 187-211.

Muzlera, J. (2011). Agricultura familiar y contratismo de maquinaria agrícola a comienzos del siglo. En: N. López Castro y G. Prividera (comps.) (2011). Repensar la agricultura familiar. Aportes para desentrañar la complejidad agraria pampeana. Buenos Aires: CICCUS Ediciones. p. 265-286.

Obschatko, E. (2009). Las explotaciones agropecuarias familiares en la República Argentina. Un análisis a partir de los datos del Censo Nacional Agropecuario 2002. 1a. Edición. Buenos Aires: Ministerio Agricultura, Ganadería, Pesca y Alimentos. Instituto Interamericano de Cooperación para la Agricultura, 2009. 68 p.

Ottmann, G., Spiaggi, E., Renzi, D. y Miretti, A. (2009). El desarrollo tecnológico en la región pampeana y su impacto socio-ambiental. El caso del sur de la Provincia de Santa Fe. Argentina. Agricultura sostenible. Vol. 5. 10pp. Agroecología. Universidad Autónoma de Chiapas. Sociedad Mexicana de Agricultura Sostenible. México.

Rosenstein, S.; Faccinini, D.; Montero, G.; Lietti, M.; Puricelli, E.; Tuesca, D.; Nisensohn, L. y Vignaroli, L. (2007). Estrategias productivas, prácticas de control y diversidad biológica: un análisis desde los sistemas de conocimiento. Revista FAVE-Ciencias Agrarias 5/6(1-2): 42-60.

Rossi, D. (2006) El contexto del proceso de adopción de cultivares transgénicos en la Argentina. Revista Agromensajes de la Facultad de Ciencias Agrarias de la UNR.Número 20.

Salembier, C., J.H. Elverdín y Meynard, J.M. (2016). Tracking on-farm innovations to unearth alternatives to the dominant soybean-based system in the Argentinean Pampa. Agro. Sustain. Dev. 36: 1. doi:10.1007/ s13593-015-0343-9

Sánchez Valduví, G.E. (2012). Manejo de malezas en lino. Evaluación de la competencia cultivo-maleza con un enfoque agroecológico (Tesis doctoral). Recuperado de http://sedici.unlp.edu.ar/handle/10915/26043.

Sarandón, S.J. (2009). Biodiversidad, agrobiodiversidad y agricultura sustentable: Análisis del Convenio sobre Diversidad Biológica. En: M. Altieri (Ed.) (2009). Vertientes del pensamiento agroecológico: fundamentos y aplicaciones. Medellín: SOCLA. Capítulo 4: 95-116.

Sarandón, S.J. (2014). El agroecosistema: un ecosistema modificado. En: S.J. Sarandón y C.C. Flores (eds.) Agroecología: bases teóricas para el diseño y manejo de agroecosistemas sustentables (Capítulo 4: 100-130). La Plata: Edulp. Recuperado de http://sedici.unlp.edu.ar/handle/10915/37280 . 
Sarandón, S.J. (2016). Potencialidades, limitaciones y desafíos para la introducción de la Agroecología en la Educación Agrícola Superior en la Argentina. El caso de la Cátedra de Agroecología de la Universidad Nacional de La Plata: Una experiencia de 16 años. Revista Agroecología, 11 (1): 47-61.

Sarandón, S.J. y Flores, C.C. (2014a). La insustentabilidad del modelo agrícola actual. En: S.J. Sarandón y C.C. Flores (eds.) Agroecologia: bases teóricas para el diseño y manejo de agroecosistemas sustentables (Capítulo 1: 13-41). La Plata: Edulp. Recuperado de http://sedici.unlp.edu.ar/handle/10915/37280 .

Sarandón, S.J. y Flores, C.C. (2014b). La agroecología: el enfoque necesario para una agricultura sustentable. En: S.J. Sarandón y C.C. Flores (eds.) Agroecologia: bases teóricas para el diseño y manejo de agroecosistemas sustentables (Capítulo 2: 42-69). La Plata Edulp. Recuperado de http://sedici.unlp.edu.ar/handle/10915/37280.

Sarandón, S.J. y Hang, G. (2002). La investigación y formación de profesionales en Agroecología para una agricultura sustentable: el rol de la Universidad. En: S.J. Sarandon (Ed.) (2002). Agroecología. El camino hacia una agricultura sustentable. La Plata: Ediciones Científicas Americanas, 2002. Cap. 23: 451-464.

Scott, D. (2011). The Technological Fix Criticisms and the Agricultural Biotechnology Debate.J. Agri. Environ Ethics, 24:207-226

Sili, M. (2005). La Argentina rural: de la crisis de la modernización agraria a la construcción de un nuevo paradigma de desarrollo de los territorios rurales. Ediciones INTA. Buenos Aires, 108 pp. Recuperado de https://ciier10.wikispaces.com/file/view/Sili+La+argentina+rural.pdf

Tamagno, L.N., Iermanó, M.J., Sarandón, S.J. y Pérez, R.A. (2014). Influencia de los saberes de los agricultores familiares pampeanos sobre las decisiones productivas y tecnológicas: su relación con un manejo sustentable. IX Congreso Latinoamericano de Sociología Rural 2014. Asociación Latinoamericana de Sociología Rural (ALASRU). México, 6 al 11 de octubre de 2014.

Tamagno, L.N., Iermanó, M.J. y Sarandón, S.J. (2015). El cultivo de soja en la producción familiar de la región pampeana: ¿Cómo y Por qué? IX Jornadas interdisciplinarias de estudios agrarios y agroindustriales argentinos y latinoamericanos. CABA, 3, 4, 5 y 6 de noviembre de 2015.

Toledo, V.M. (2005). La memoria tradicional: la importancia agroecológica de los saberes locales. LEISA Revista de Agroecología, p.16-19. Recuperado de http://www.edtech.ku.edu/new/lessons/english/conservation/media/La _memoria_tradicional.pdf

Tsakoumagkos, P. (2009). Tecnología y pequeña producción agropecuaria en la Argentina: una caracterización basada en el censo nacional agropecuario 2002 y en estudios de caso / P. Tsakoumagkos y González M. C.; coord.: Pedro Tsakoumagkos. Ed. Buenos Aires: Ministerio de Agricultura, Ganadería y Pesca, 2009. 304 p. Recuperado de http://www.ucar.gob.ar/images/publicaciones/Tecnolog\%C3\%ADa\%20y\%20Peque\%C3\%B1a\%20Produ cci\%C3\%B3n.pdf

Viglizzo E.F., Frank F.C., Carreño L.V., Jobbagys E.G., Pereyra E., Clatt J., Pincen D. y Ricard F. (2011). Ecological and environmental footprint of 50 years of agricultural expansion in Argentina. Global Change Biology, 17:959-973 